\title{
XVIII.
}

\section{Ueber primäre und secundäre Natur der bei der Mehrzahl von Deformitäten betheiligten Organe.}

Eine Beleuchtung der von Dr. Leopold Dittel äber den betreffenden Gegenstand veröffentlichten Ansichten.

Von Dr. Eulenbarg zu Berlin.

(Nachtrag zu XVII.)

Wenn sich der Irrthum häufig wiederholt, so mufs auch die Wahrheit oft wiederholt werden. Jeder redliche Autor trägt nun zwar die Ueberzeugung in sich, dafs seine Ansichten die Wahrheit enthalten. Dennoch kann er irren. Wenn ich nun dies auch in Bezug auf mich vollkommen zugestehe, so kann ich doch der Bekämpfung derjenigen Ansichten auf dem Gebiete der Orthopädie, welche ich nach der mir möglichen Einsicht für irrig erkannt habe, so lange nicht widerstehen, als ich nicht von dem Gegentheile überzeugt worden bin. Der Leser mag über die entgegenstehenden Ansichten aus unbefangener Prüfung sein Urtheil fällen. Wie es auch ausfalle, wenn auch meiner Ansicht entgegen, immer würde die Erörterung nicht ganz unfruchtbar geblieben sein.

Bereits vor einigen Jahren hat Dr. Leopold Dittel zu Wien aus Sectionen gewonnene Beobachtungen über einige Deformitäten mitgetheilt, welche mich und gewils Jeden, der das dringende Bedürfnils solcher pathologisch-anatomischen Befunde anerkennt, im hohen Grade interessiren. Es wird dem Werthe der von Dittel gefundenen Thatsachen nicht Abbruch 
thun, wenn nicht alle Leser den vom Autor daraus gezogenen Theorieen beistimmen. Je öfter und sorgsamer ich seine Arbeit studirt habe, desto mehr hat dieselbe dazu beigetragen, mich in meiner von der des Autors vielfach abweichenden Ansicht über die primären und secundären Verhältnisse der in Rede stehenden Deformitäten zu bestätigen. Um diese Ansichten zu motiviren, mufs ich mir gestatten, in der Kürze die bezüglichen Stellen aus der Dittel'schen Mittheilung in wörtlicher Anführung voranzustellen.

Die Arbeiten Dittels finden sich in der Zeitschrift der k. k. Gesellschaft der Aerzte zu Wien. Sie handeln

1. Ueber das frische Präparat eines Klumpfufses (Pes varus) zweiten Grades; im April(III.)-Heft. 1851.

2. Ueber das frische Präparat eines Pes equinus zweiten Grades; im Juni(VI.)-Heft. 1851.

3. Ueber das frische Präparat eines Pes valyus; im Mai(V.)Heft. 1852.

4. Ueber Scoliose; im Mai(V.)-Heft. 1853.

Ad 1. Dittel sagt S.280.: "Es wird wohl Niemandem einfallen, jene Veränderungen, die sich durch Zustände der Verlängerung, Dehnung, Zerrung und Erschlaffung kund geben, anders als secundäre zu bezeichnen. Dagegen halte ich es für sehr schwer, unter den verkürzten, verdichteten und gespannten Bändern und den auf dieselbe Art veränderten Muskeln heraus zu finden, ob die Bänder- oder ob die Muskelveränderung als primäre zu bezeichnen sind."

Aus den 10 Nummern des Schlufsresumés hebe ich folgende von Dittel angeführte Facta und Folgerungen hervor:

$S u b$ 2. „Alle an der Convexität der Curve liegenden Weichtheile sind gedehnt, verlängert, erschlafft, schlechter genährt, theilweise - einige Muskelbündel - in Fett verwandelt."

Sub 3. „Die an der Concavität der Curve liegenden Weichtheile sind verkürzt, gespannt und besser genährt." .

Sub 7. „Die Bänder, die die Deformität mitbedingen, sind die am inneren Fufsrande liegenden und ganz besonders das 
Ligam. delloiderm und das Lig. calcaneo-naviculare interosseum."

Sub 9. Die bezeichneten Bänder scheinen alle Ansprüche zu haben auf den primären Ursprung ihrer Erkrankung.

Ad 2. Bei Erörterung der Frage, welchen der betheiligten Organe in genetischer Beziehung die Bedeutung primärer oder secundärer zukomme, gelangt Dittel hier (beim Pes-equinus) zu dem Schlusse, dafs die Veränderungen in den Knochen secundärer Natur sind.

In Betreff der primär ursächlichen Theile stellt er einen Unterschied auf zwischen erworbenem und angeborenem Pes equinus. Bei ersterem ist die verkürzte Achillessehne und die Aponeurosis plantaris das primär ergriffene Organ. Bei dem angeborenen Pes equinus will er diesen noch die Verkürzung und Verdickung des Lig. calcaneo-cuboideum plantare und des Lig. calcaneo-naviculare plantare als primäre ursächliche Momente mit eingereiht wissen.

In Betreff des Antheils der Muskeln findel sich nur folgende Bemerkung vor: „Die gesammte Muskulatur der Wade war im Vergleich zu der des rechlen Unterschenkels bedeutend schwächer entwickelt. Dieser atrophische Zustand der Wadenmuskeln, der in physiologischen Widerspruch zu stehen scheint mit dem erschwerten Gehen und der 'somit gesteigerten Innervation (?) dieser Muskeln, ist dadurch zu erklären, dafs der dynamische Prozels ein abgeschlossener ist, die Muskeln schon lange das Maximum ihrer Contractionen erreicht haben, über welches hinaus sie weder weiter thätig, noch durch ihre Antagonisten aus ihrem Zustande gebracht werden können, indem auch die unnachgiebigen Aponeurosen sich bereits dem neuen organischen Verhältnisse angepafst haben."

Leider hat Dittel es hier versäumt, die Beschaftenheit der zur Difformität in Beziehung stehenden Muskeln an der convexen und concaven Seite der Curve rücksichtlich ihrer Structurverhältnisse zu vergleichen, wie er dies beim $P$ es varus und noch ausführlicher beim folgenden Fall, dem Pes valgus, ge-

Archiv f, pathol, Anat. Bd. IX. Heft 4 . 
than. Aus diesem Grunde wähle ich diesen behufs des vorliegenden Zweckes zur ausführlichen Besprechung.

Ad 3. Das von Dittel untersuchte Präparat des Pes valgus rührte von einem 30jährigen Manne her, dessen linker Fufs normal war. Dittel benutzt daher die Gelegenheit, sowohl die Muskeln des kranken Fufses unter sich, als auch mil denen des gesunden zu vergleichen. Hierbei dringt sich ihm, wie er 1. c. S. 405. bemerkt, in Bezug auf die Form- und Texturveränderungen eine gewisse Gesetzmälsigkeit auf, die man an anderen Deformitäten wiederfindet. In Vergleich mit dem gesunden Fufse ist die Muskulatur am kranken durchaus nirgends so voluminös entwickelt, vielmehr überall dünner, schmächtiger, abgeflacht, blafsroth, gelblich, verfettet.

Beim Vergleich der betreffenden Muskeln des kranken Fufses unter sich resumirt Dittel 1. c. S. 407.: „Wir haben sowohl an den verkürzten, als an den verlängerten und gezerrten Muskeln die Zeichen der Atrophirung und fettigen Degeneration gefunden. Jedoch müssen wir zugeben, dafs diese Zeichen mehr entwickelt waren an den gedehnten, als an den verkürzten, d. i. contracten Muskeln, und zwar mil solcher Consequenz, dafs die Unterschiede nicht selten an den verschiedenen Köpfen eines und desselben Muskels a uffallen." Man kann aus dieser Thatsache, bemerkt Dittel, den Schluls ziehen, dals der Zustand der Ruhe eines Muskels seine fettige Degeneration bedinge und dafs noch insbesondere seine Ruhe im Zustande der Ausdehnung die Atrophirung und feltige Entartung desselben begünstige.

Ich gedenke nachzuweisen, dals dieser Befund noch zu ganz anderen sehr nahe liegenden, erweislichen und therapeutisch wichtigen pathogenetischen Aufklärungen führen murs. Bevor ich dahin gelange, muls ich die weiteren Ansichten des Autors über die Entstehung des Valgus anführen. Dittel sagt 1. c. S. 417.: „Wenn man das combinirte Astragalo-naviculocalcanear-Gelenk als den Ausgangspunkt der Valgus-Deformität annimmt, so kann man freilich wohl die Frage um den 
lelzten Grund derselben um einen Posten weiter vorschieben." Da verlälst ihn aber die anatomische Basis und er „überweist die Frage dem physiologischen (Grübler) Forscher". Soll ein Leiden der Nervencentra im Embryonalleben und somit eine krankhafte Innervation die erste Veranlassung zu einer vereinzelten Muskelcontraction abgeben, so will er dafür im Leben kein Analogon finden. Die zufällige Lage des Embryo anzuschuldigen, sei leichter und bündiger abfertigend, da sich unter ähnlichen Verhältnissen die erworbenen Contractionen nach der Geburt entwickeln. Unter solchen Erwägungen folgert nun Dittel: „Er könne sich nicht leicht denken, dafs eine der wichtigen Veränderungen am angeborenen Valgus als secundär zu betrachten sei." (Demnach wäre also Alles primär.) Dittel glaubt vielmehr annehmen zu müssen, „dals, einmal der Keim zur Deformität gegeben (ich bitte den Leser auf diese Captivirung wohl zu achten), sich diese in derselben ebenmälsigen Harmonie aller contribuirenden Theile ausbilde, wie es in der physiologischen Entwickelung der Fall ist."

Dieser von Dittel als vorhanden angenommene Keim zur Deformität ist ja eben das, was als das primär dieselbe bedingende ursächliche Moment zu erforschen ist. Ein solches vermissen wir bei Dittel denn auch. Die I. c. S. 406. aufgeführte Erklärung präsumirt eben eine vorhandene Deformität, anstatt ihr Entstehen zu deuten. Er sagt dort: „dafs der Fufs in dem Astragalo-naviculo-calcaneal-Gelenke eine Drehung macht, wodurch der äufsere Fufsrand nach aufwärts und zugleich etwas nach rückwärts tritt." Diese Drehung ist ja doch aber schon die Deformität selbst; und anstatt ihre Entstehung zu erklären, sagt Dittel weiter: „dadurch werden die Insertionen der am äufseren Fufsrande gelegenen und der zunächst befindlichen (?) Muskeln ihrem Ursprung näher gerückt. Umgekehrt ist das Verhältnifs am inneren Fufsrande, welcher nach abwärts tritt. Diesen neuen mechanischen Verhältnissen passen sich also die Weichtheile vollkommen an. Die an dem inneren Fulsrande liegenden sind daher (?) gezerrt, gedehnt; die am äufseren Fufsrande liegenden haben gegen- 
wärtig eine kürzere Distanz zu durchwandern und sind daher verkürzt. Versucht man dem Fulse seine normale Stellung zu geben, so werden die am äufseren Fufsrande liegenden Muskeln und Sehnen in Spannung versetzl. Man hat diese Eigenschaft als einen Beweis der Muskelthätigkeit angesehen und sie unter dem Namen der activen Muskelretraction als Ursache der Deformität angeführt."

Diese Ansicht sucht Dittel in vier Zeilen zu widerlegen und fährt dann weiter fort: „Man könnte hier allerdings die Frage aufstellen, warum der in sogenannter activer Retraction begriffene Muskel sich nicht mindestens bis zu seiner normalen Länge ausdehnen läfst. Darauf läfst sich nur antworten, dafs die Länge des Muskels bestimmt ist durch die Stellung jenes Gliedtheils, an welcher sich sein Ursprung befindet, zu jenem, an welchem sich sein Endpunkt inserirt. Wenn nun diese 2 Punkte einander näher rücken, so wird der Muskel kürzer werden, und wenn nun diese 2 Punkte in ihrer Annäherung unausgesetzt beharren, so wird der Muskel auch jene Organisation eingehen; die seinem neuen verkürzten Zustande entspricht. Dann wird er zum eigentlichen - nicht contrahirten, sondern contracten, d. i. verkürzten Muskel. Auf diese Art sehen wir oft vor unseren Augen eine Contractur im Kniegelenke sich ausbilden, wern bei der Entzündung des Kniegelenks der Unterschenkel beständig gebeugt erhalten wird."

Diese wörtliche Mittheilung der Dittel'schen Ausführung wird jedem etwaigen Vorwurfe begegnen, dals ich die Ansicht des Autors irgendwo irrthümlich dargestellt hätte. Um diese Ansichten zu widerlegen, kehre ich zu dem von Dittel präsumirten Ausgangspunkte, der vorerwähnten Drehung des Fufses zurück.

Was heifst denn das: „der Fuls macht in dem Astragalonaviculo-calcaneal-Gelenk eine Drehung und dadurch werden die Insertionen der am äufseren Fufsrande gelegenen Muskeln ihrem Ursprunge näher gerückt u. s. w."? Diese Drehung des Fulses, die Dittel vorweg annimmt, ist ja eben derjenige Theil der vorhandenen Deformität, deren Entstehungsursache physio- 
logisch erklärt werden soll, diese Drehung ist ja die erste $A b$ weichung von der physiologischen Form und Function des Fufses, die Deformität selbsí. Dittel bleibt uns daher die Beantwortung der Frage schuldig, wodurch diese Drehung entstehe, und ist gewillt, unter dem Vorwande, dafs ihn die anatomische Basis hier verlasse, dieselbe dem physiologischen (Grïbler) Forscher zu übergeben.

Ich meine trotzdem, dafs man allerdings aus der Physiologie die Endursachen der Abweichungen von den physiologischen Functionen aus pathologisch-anatomischen Befunden zu erkiären suchen müsse. Die von Dittel und auch von anderen vor ihm vorgefundenen Facta sprechen hier so deutlich, dafs man nicht nölhig hat, sich in "Grübeleien" zu verlieren, sondern nur, auf dem ehrenvollsten Boden der physiologischen Forschung stehen zu bleiben.

Die Physiologie lehrt uns aber, dafs ein Knochen und Alles, was zu den passiven Bewegungsorganen eines Gelenkes gehört, sich nicht bewegt, sich nicht spontan dreht, dafs also Insertionspunkte von Muskeln nicht von selbst aneinander rücken, sondern dafs die Knochen und die ihnen zugehörigen Ansatzpunkte der Muskeln bewegt werden. Die Organe, die diese Thätigkeit vollziehen, sind einzig und allein die Muskeln. Man muls daher, um eine richtige Anschauung des pathogenetischen Vorganges zu haben, die hier erwähnten Dittel'schen Erläuterungen vollkommen umkehren. Nicht die Muskeln passen sich den neven mechanischen Verhältnissen der Knochen an, sondern die anomale Function der Muskeln erzeugt erst diese neuen mechanischen Verhältnisse. Gerade die Knochen, Knorpel, Ligamente und Aponeurosen sind es, welche sich mechanisch der Muskelthätigkeit unterwerfen müssen.

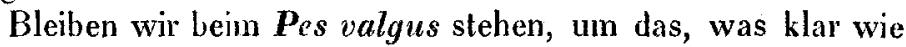
die Sonne ist, dennoch nachzuweisen, da doch ein Mal solche tief gewurzelte Irrthümer immer wieder und wieder vorkommen.

Die am inneren Fufsrande liegenden Muskeln sind nicht "daher gedehnt", weil die newen mechanischen Verhältnisse, die Drehung des Fufses, es bedingen, sondern ungekehrt, weil 
diese Muskeln erschlafft sind und deshalb in ihrer physiologisch normalen Function eine verminderte Energie entwickeln, werden sie Veranlassung zu den in die Erscheinung tretenden neuen abnormen mechanischen Verhältnissen, der Drehung des inneren Fufsrandes nach abwärts, welche man unter der mit Pes valyus bezeichneten Deformität selbst versteht. Die Drehung ist die nothwendige Folge einer vorhandenen Functionsstörung, ist pathognomonisches Symptom, wie die abnorme Stellung des Bulbus beim Strabismus die Folge von perverser Muskelaction ist. Gerade in diesen von Dittel unbegreiflicherweise gar nicht gewürdigten an der Convexilät dieser und aller anderen ähnlichen Deformitäten befindlichen Muskeln, welche er bei der Autopsie gedehnt, erschlafft, atrophirt gefunden hat, liegt der Ausgangspunkt der Deformität, von welchem aus alle anderen mit der Dauer der Deformität sich immer mehr entwickelnden Veränderungen in ungezwungenster Weise erklärt werden können.

Es sind daher ferner die am äufseren Fufsrande liegenden Muskeln nicht "deshalb verkürzt", wie Dittel behauptet, dafs sie, "sich den neuen mechanischen Verhältnissen anpassend, eine kürzere Distance zu durchwandern hab en", sondern sie erzeugen diese kürzere Distance, sie nähern die Knochenpunkte, an welche sie sich inseriren, indem und weil sie sich verkürzen. Ihre Verkürzung ist eine Nothwendigkeit, weil ihre am inneren Fufsrande liegenden und wirkenden Antagonisten erschlafft sind; ihre Verkürzung erfolgt nicht nur obgleich, sondern weil sie gesund sind. Diese Verkürzung ist allerdings, genau genommen, nicht eine active, insofern sie nicht vom Willen intendirt jst, sondern sie ist eine unausbleibliche Folge des automatischen Muskeltonus, wie sie unsere gediegensten Physiologen, Müller, Hyrtl, Valentin u. A. entschieden anerkennen. Sie muls stets bei allen gesunden Muskeln eintreten, sobald ihre Antagonisten aus irgend welcher pathischen Ursache in ihrer Function gehemmt oder nur geschwächt sind. Sie kann daher als ein physiologisches Gesetz betrachtet werden. 
Wovon bei den der convexen Curve angehörigen gedehnten Muskeln die Verminderung oder Hemmung der einseitigen Muskelfunction abhängt, ist eine weiter zu erörternde Frage, die ich anderwärts ausführlich besprochen habe. Ich betrachte als ihre Veranlassung die meistens von exsudativen Prozessen in der Nähe der motorischen Spinalwurzeln oder in den Nervencentris abhängige anomale Innervation. Doch können auch Störungen im peripherischen Nervenapparat die Veranlassung sein, wie solche z. B. am offenkundigsten beim Nervus facialis beobachtet werden.

Dieses wichtige der physiologischen Anschauung von der antagonistischen Muskelthätigkeit entnommene pathogenetische Moment läfst der geschätzte Autor unbegreiflicher Weise ganz unberücksichtigt. Niemals spricht er auch nur die Vermuthung aus, dafs die an der convexen Deformitätcurve gelegenen erschlafften und gedehnten Muskeln in irgend welcher ursächlichen Bexiehung stehen. Ja beim Varus l. c. S. 280. sagt er geradezu: „Es wird wohl Niemandem einfallen, jene Veränderungen, die sich durch Zustände der Verlängerung, Dehnung, Zerrung und Erschlaffung kund geben, anders als secundäre zu bezeichnen." Nun ich bekenne, dafs ich völlig entgegengesetzter Ansicht bin. Auf der concaven Seite aber, wo es Dittel schwer wird, sich für die primäre ursächliche Natur der verkürzten Bänder oder Muskeln zu entscheiden, fällt seine Wahl auf die Bänder. Warum Dittel sich zu Gunsten dieser physiologisch der Activität der Muskeln untergeordreten Organe entscheidet, sagt er nicht, und ist auch gar nicht zu begreifen. Eben so mechanisch will Dittel die Frage lösen, warum der in sogenannter activer Retraction befindliche Muskel sich nicht mindestens bis zu seiner normalen Länge ausdehnen läfst. Es soll dies nur daher rühren, dafs die Länge des Muskels bestimmt sei durch die Stellung der Insertionspunkte. Man ist wirklich bei solcher Deutung versucht, sich Aufklärung zu erbilten, welche Function denn die Muskeln haben, wenn sie nicht die Bewegung vermitteln. Eine active Retraction existirt aber meiner Ansicht nach gar nicht. Es giebt nur 
eine active Contraction; denn unter Retraction verstehen wir eine organisch gewordene permanente Contraction. Wegen dieser organischen Texturveränderung läfst sich ein contrahirter Muskel nicht ausdehnen. Von der Richtigkeit dieser Behauptung kann man sich z. B. am Sternocleidomastoideus beim Caput olstipum aufs Unzweideutigste überzeugen. Oder möchte Dittel hier auch behaupten, dafs die Länge des Muskels bedingt ist durch die Annäherung des Processus mastoideus an das Sternum und das Sternalende der Clavicula? Ist es denn hier nicht unzweifelhaft, dals gerade die Annäherung dieser beiden Insertionspunkte bedingt ist durch die Verkürzung des Muskels? Durch die Tenotomie fallen die Insertionspunkte ja sofort ebenso auseinander, wie sie an der entgegengesetzten Seite sich nähern, weil der ihre Distance bestimmende Muskel nun, nach Entfernung der Retraction seines Antagonisten wieder in der Lage isl, sich zusammen zu ziehen.

Ganz ebenso verhält sich's beim Pes valgus, varus, equinus etc.

Es ist aber gar nicht einmal stets der Fall, dafs die verkürzten Muskeln sich nicht mehr oder weniger bis zu ihrer normalen Länge ausdehnen lassen. Zahlreiche Fälle von $\boldsymbol{P} e s$ valgus, Pes equinus, Hackenfuls u. s. w. habe ich gesehen, in denen diese Ausdehnung passiv mit Leichtigkeit zu bewirken war. Freilich ist dies in der Regel nur bei Kindern der Fall und bei nicht allzulanger Dauer der Deformität. Dittel's Präparat des Valgus war von einem 30jährigen Manne, das des Pes varus gar von einem 40jährigen Individuum. Wann der Pes valgus entstanden sei, ist nicht angegeben. Aus einigen am Ende der Abhandlung befindlichen Andeutungen über das Embryonalleben ist der Schlufs gerechtfertigt, dafs die Deformität eine angeborene war. Nun bemerkt Dittel aber selbst l. c. S. 407.: „wenn ein Muskel unausgesetzt in der verkürzten Lage beharrt, so wird derselbe auch jene Organisation eingehen, die seinem verkürzten Zustande entspricht." Demnach wird man wohl zugestehẹ können, dals beim Pes valgus die am äufseren Fufsrande befindlichen verkürzten Muskeln, wenn sie Anfangs auch noch so dehnbar waren, wohl in weniger áls 
30 Jahren die erwähnten Veränderungen eingehen können, um retrahirt oder, wie Dittel und $A$. es bezeichnen, contract zu sein.

Das Bestehen einer Retraction an einem so veralteten Pes valgus beweist daher nichts für die primäre Retraction der verkürzten Muskeln, am allerwenigsten aber für die Bänder, die in ihrer gänzlichen Passivität noch eher den Texturveränderungen verfallen, als die Muskeln.

Dagegen finden sich in Dittel's Beschreibung des Befundes Facta, welche den Autor zu der Ansicht hätten bestimmen müssen, dafs die an der convexen Curve, dem inneren Fufsrande, gelegenen Muskeln die primär erkrankten waren. Dittel führt l. c. S. 407. an, „dafs die Zeichen der Atrophirung und fettigen Degeneration mehr entwickelt waren an den $\mathrm{ge}$ dehnten als an den verkuirzten, d. i. contracten Muskeln, und zwar mit solcher Consequenz etc." (s. oben). Diesen Thatbestand benutzt Dittel zu nichts weiler, als um uns ein neues Gesetz für die Bedingungen zur fettigen Degeneration aufzustellen, indem er die völlig unmotivirte Theorie entwickelt, dafs die Ruhe (Unthätigkeil) eines Muskels im Zustande der Ausdehnung die Atrophirung und fettige Degeneration desselben vorzugsweise begünstige. Es ist aber mit diesem neuen Gesetze Dittel's eben so wenig gewonnen, als mit dem von Guérin aufgestellten, wonach die retrahirten Muskeln zur Versehnung, die gedehnten zur Verfettung übergehen sollen. Mich dünkt, man könne die vorgefundene Thatsache physiologisch leicht erklären, ohne zu so practisch unfruchtbaren Theorieen Zuflucht nehmen zu müssen, abgesehen davon, dals gar kein vernünftiger Grund vorhanden ist, weshalb ein unthätiger gedehnter Muskel mehr zur Atrophirung und Verfettung disponirt sein solle, als ein unthätiger verkürzter. Nicht in der Dehnung oder Verkürzung liegt der Grund dieser Erscheinung, sondern im pathologischen Zustande selber. Die in den gedehnten Muskeln vorwaltend angetroffene Atrophirung können wir als Folge und Beweis betrachten, dafs sie die ursprünglich primär erkrankten waren. Die Leitung des sie beherrschenden motorischen Nerven war durch pathische 
Verhältnisse unterbrochen. Dadurch war in erster Linie die Unthätigkeil der Muskeln bedingt. Mit der Unthätigkeit_ist die verminderte Zuströmung arteriellen Blutes und der verminderte Stoffwechsel eng verbunden. Daher die Hemmung der Ernährung und die Rückbildung der Muskeln in die niederen Organisationsstufen. Die an der Concavität gelegenen verkürzten Muskeln dagegen sind deswegen weniger zur Atrophie disponirt, weil eben bei ihnen ein wesentliches Moment zu dieser Desorganisation fehlt, nämlich ihre ursprüngliche Erkrankung. Ihre centrifugale Innervation ist ungestört. Weil sich dies so verhält, weil sie gesund sind, verkürzen sie sich vermöge des automatischen Muskeltonus um ében so viel, als ihre der normalen Innervation beraubten Antagonisten sich krankhaft ausdehnen. Wäre die Innervation der verkürzten Muskeln in gleicher Weise erloschen, wie die der Antagonisten, so könnte die Deformität gar nicht zu Stande kommen, wie wir dies bei einer vollständigen Paralyse oder einer gleichniälsigen Schwäche der gesammten Muskulatur eines Gliedes sehen. Tritt in Folge von Muskelparalyse eine Deformität ein, so ist dies immer ein Zeichen, dafs die Antagonisten der gelähmten Muskeln ihrer vitalen Function keinesweges ganz beraubt sind.

Von der Richtigkeit dieser Ansicht kann man sich im Anfangsstadium dieser Deformitäten vollkommen und später wenigstens so lange genügend überzeugen, als die Knochen, Knorpel, Ligamente und Aponeurosen und mit ihnen selbst die verkürzten Muskeln nicht secundär allzubedeutende Veränderungen der Form und Textur erlitten haben. Man bringe zu diesem Zwecke das deforme Glied passiv in die normale Stellung, indem man die verkürzten Muskeln ausdehnt, und gebe dem Kranken auf, diese wieder zu contrahiren. Er vermag dies vollkommen. Dagegen fehlt ihm diese Einwirkung auf die an der Convexität befindlichen gedehnten Muskeln, je nach dem Grade der gestörten Nervenleitung, entweder vollkommen oder theilweise. Darin liegt zugleich der alleinige Grund, dals der unfreiwillig contrahirte Muskel sich nicht durch den Willenseinflufs des Kranken auszudehnen vermag. 
Es fragt sich nun, woher es komme, dafs auch diese an der Concavität gelegenen, von mir als gesund bezeichneten Muskeln dennoch atrophiren, wenn auch in entschiedenster Consequenz stets weniger als ihre kranken Antagonisten? Diese Thatsache, dünkt mich, ist physiologisch so zu erklären: die bei einer in Folge von Muskelparalyse eingetretenen Deformität an der concaven Curve gelegenen ursprünglich gesunden Muskeln sind, wie wir gesehen haben, durch die Unthätigkeit ihrer Anlagonisten gezwungen, selbst dauernd in unthätigem Zustande, in Contraclion, zu verharren. Dadurch fehlt ihnen ihr physiologisches Thätigkeitsgebiet, die Bewegung. Jedes Organ, das seiner physiologischen Thätigkeit, aus welchem Grunde es immer sei, dauernd verlustig geht, mufs nothwendig Einbufse erleiden an seiner physiologischen Integrität. Wir sehen diesen Grundsatz überall bestätigt und ganz besonders auch beim ganzen Locomotionsapparat. Muskeln, welche eine Beschränkung der Bewegung erleiden, verlieren ebenso an Energie und Nutrition, als sie umgekehrt an diesen Eigenschaften gewinnen durch ein vernünftig gesteigertes Maafs der Bewegung.

Das ist also der Grund, weshalb auch die an der concaven Seite gelegenen zwar gesunden, aber in ihrer Function gehinderten Muskeln zwar atrophiren, aber niemals in dem Grade, als ihre durch ursprüngliche Krankheit unthätigen Antagonisten.

Die von Manchen ausgesprochene Ansicht, dafs dieselben, eben weil sie verkürzt sind, sich in Ueberthätigkeit befinden und deshalb hypertrophisch sein müfsten, ist völlig unbegründet. Denn zur Thätigkeit eines Muskels gehört nicht blofs Contraction, sondern auch Expansion. Die permanente Verkürzung ist ebenso unzureichend für die Integrität des Muskellebens, wie die permanente Dehnung.

Bei Gelegenheit der Besprechung des Pes equinus-Präparats sagt Dittel: „Der atrophische Zustand der Wadenmuskeln, der im physiologischen Widerspruche zu stehen scheine mit dem erschwerten Gehen und der somit gesteigerten Innervation dieser Muskeln, erkläre sich daraus, dafs der dynamische Prozefs ein abgeschlossener sei, dafs die Muskeln schon lange 
das Maximum ihrer Contractionen erreicht haben, über welches hinaus sie weder weiter thätig, noch durch ihre Antagonisten aus ihrem Zustande gebracht werden können."

Diese Auffassung des geschätzten Verfassers ist sicherlich unrichtig.

Beim Pes equinus sind die Wadenmuskeln die verkürzten, der concaven Curve angehörigen, die am Fulsrücken gelegenen die krankhaft gedehnten. Es ist nun aber gar nicht wahr, dafs die Wadenmuskeln, weil ein Pes equinus besteht, sich im Zustande erhöhter Innervation befinden. Ja sie sind vielmehr, wenn auch in ihnen die Nervenleilung ungestört ist, bei ihrem durch die Unthätigkeit ihrer Antagonisten bedingten unfreiwilligen Verkürzungszustande an der Entwickelung der ihnen zukommenden Innervation und Thätigkeit im hohen Grade beschränkt. Der mit dieser oder einem Pes varus, Pes valgus u. s. w. behaftele Kranke ist an dem normalen Gebrauche des Fulses gehindert. Dieses Hindernils betrifft zwar vorzugsweise die paralysirten Muskeln, erstreckt sich aber auch, wie oben nachgewiesen wurde, mehr oder weniger auf die Antagonisten, das sind beim Pes equinus die Wadenmuskeln. Bei dieser Deformität ist fast die ganze Muskulatur des Unterschenkels aufser Gebrauch. Der Kranke bedient sich des Unterschenkels gewissermafsen nur wie eines Stelzfulses mittelst der Muskulatur des Oberschenkels. Diese letztere ist es daher, nicht die Muskulatur des Unterschenkels, welche sich in einem erhöhten Innervationszustande befindet. Und diese vermehrte Innervation ist auch in der Muskulatur des Oberschenkels sichtbar. Sie documentirt sich hier durch eine aufserordentliche Nutrition derselben, wie ich dies in zahlreichen Fällen stets beobachtet habe.

Hiernach erklärt sich nun von selbst die durch den $\mathrm{Ob}$ ductionsbefund ermittelte Thatsache, dafs die an der Convexität der Curve gelegenen Muskeln consequent mehr atrophirt und fettig degenerirt gefunden wurden, als die verkiirzten an der Concavitäl gelegenen. Denn während diese bei ungestörter motorischer Nervenleitung unthätig sind, allein aus mangelnder anlagonistischer Erregung, verfallen jene gedehnten an der 
Convexitäl gelegenen der Unthätigkeit aus einem direct sie betreffenden pathischen Zustande, aus anomaler motorischer Innervation.

In jenen selteneren Fällen, wo die Verkürzung_der an der concaven Curve gelegenen Muskeln primär in Folge krankhafter Nervenleitung entstanden ist, und die gesunden Antagonisten secundär in den gedehnten Zustand versetzt werden, mufs sich nothwendig das Verhältnifs der Texturveränderung umkehren. Denn von der Dehnung oder Verkürzung eines unthätigen Muskels kann das Verfetten und Atrophiren unmöglich bedingl sein, wohl aber von dem unterbrochenen Einflufs des motorischen Nervenapparates. Je länger nun jene Mifsverhältnisse bestehen, wodurch die Muskeln in ihrer physiologischen Function gehindert sind, desto mehr wird die Ernäbrung derselben leiden, desto mehr werden sie der Verfettung und Atrophirung verfallen.

Dafs selbst nach 30jährigem Bestehen solchèr physiologischen Störung die Autopsie noch einen scharf ausgeprägten Unterschied in der Texlur der besser erhaltenen verkürzten, gegenüber den mehr atrophirten und verfetteten gedehnten Muskeln darthut, darin erblicke ich nur ein bestätigendes Moment mehr für die Richtigkeil meiner Ansicht.

Dittel findet diese Muskulaturverhältnisse auch beim Pes varus durch den anatomischen Befund bestätigt (s. l. c. S.281.); beim $\boldsymbol{P}$ es equinus vermissen wir indefs der speciellen Erwähnung derselben. Dagegen sagt Dittel unter Pes valgus l.c. S. 407.: "Bei Scoliosen ist die die seitliche Bewegung der Wirbelsäule vermittelnde Muskulatur sowohl an der convexen, als an der concaven Seite der Wirbelsäule minder kräftig entwickelt; aber ganz besonders sind die in der Tiefe der Concavität liegenden inehr fettig degenerirt, als die entgegengesetzten."

Wenn ich nun die Richligkeit dieses Befundes so wenig in

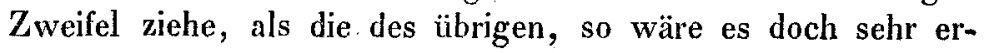
wünscht gewesen, wenn Dittel die fettig degenerirt gefundenen Muskeln einzeln namhaft gemacht hätte. Denn die Ansichten über die Function der Muskeln, welche die seitliche Beugung 
des Rückgrats vermitteln, sind keinesweges übereinstimmend. Dazu kommt, dafs dem Dittel'schen Befunde andere direct entgegenstehen, z. B. der Cruveilhier's, welcher bei Scoliose die Muskeln an den Convexitäten verringert und bleicher fand.

Uebrigens verfährt Dittel mit der pathogenelischen Auffassung der Scoliose der der Gliederdeformitäten ganz analog. Er sagt ungefähr: „Wenn die Wirbelșäule eine Kîümmung eingeht, so werde irgend ein bestimmter Wirbel sich in der gröfsten Concavität der Krümmung befinden; dieser Wirbel sei der Ausgangspunkt der Scoliose, von welchem alle übrigen Veränderungen hervorgehen." Wodurch aber die Wirbelsäule zu einer Krümmung veranlafst werde, das ist nicht gesagt, namentlich nicht, dafs eine anomale Function im activen Bewegungsapparate, in den Muskeln, zur seitlichen Beugung des Rückgrats etwas beitrage. Um so auffallender ist es, dafs Dittel l. c. S. 417. in Betreff der Stellung der Schultern Folgendes bemerkt: „Auf die Richtung des oberen oder unteren Winkels des Schulterblattes hat auch der Levator scapulae, M. rhomboideus et cucullaris Einflufs. Vom Serratus antic. major glaube jeh nicht, dafs er influirt, weil er so unmittelbar auf den Rippen anliegt, dafs es in Betreff der Schulter gleich ist, ob sie über ihm oder unmittelbar auf den Rippen ruht." Ich bekenne, dafs ich das hier vom Serratus antic. major Gesagte nicht zu verstehen vermag. Wie er sich den Einfluls der genannten Muskeln auf die Schulterdeformität denkt, erörtert der Autor lèider nicht. Weshalb aber die Schulterblätter dem Einflufs der Mưskeln bei einer Lageveränderung unterworfen seien, während es die einzelnen Glieder der Wirbelsäule nicht sein sollen, vermag ich nicht einzusehen. Ich für meinen Theil vindicire auch hier den an der convexen Curve gelegenen Muskeln den primären ursächlichen Antheil, während ich alle übrigen Erscheinungen, die Muskelverkürzung an der concaven Curve mit eingeschlossen, für secundär halte.

Zahlreiche an Scoliolischen gemachte Obductionen von Prof. Günther, Maisonneuve, Pelletan, Ouvrard, Martin de St. Ange, Cruveilhier stimmen darin überein, dafs die 
Muskeln an den Concavitäten nicht retrahirt, ja gar nicht gespannt gefunden wurden, sondern weich und nur zusammengefaltet. Daraus folgt aber unbedingt, dafs in diesen Muskeln kein Hindernifs für die Gradrichtung der Wirbelsäule besteht, da sie ihrer Beschaffenheit nach völlig ausdehnbar waren (s. Werner's Reform der Orthopädie. Berlin 1851. S. 98 u. ff.). Es folgt daraus ferner, ich will nicht sagen unbedingt, doch mit grofser Wahrscheinlichkeit, dafs dieselben nur dadurch verkürzl und zusammengefaltet waren, dafs ihre Antagonisten in primärer Weise unthätig waren und dafs sie sich um ebenso viel ausgedehnt haben würden, als die letzteren die Fähigkeit, sich zu contrahiren, wieder erlangt haben würden. Dagegen unterliegt es keinem Zweifel, dafs die Herstellung der antagonistischen Muskelthätigkeit bei der Scoliose, wie bei den anderen in Rede stehenden Deformitäten, zur Herstellung der normalen Form nicht mehr ausreicht, wenn die bei langer Dauer der Deformität eingetretenen Veränderungen im ligamentösen, knorpeligen und knöchernen Apparate als unbesiegbares Hindernils der momentanen Herbeiführung der normalen Form entgegentreten.

Diese im passiven Locomotionsapparate der in Rede stehenden Deformitäten vorkommenden Veränderungen halte ich nun in der gröfseren Zahl der Fälle für secundärer Natur.

Um jedoch nicht mifsverslanden zu werden, verwahre ich mich hier ausdrïcklich gegen den Verdacht, als ob ich die Existenz jener Deformitäten leugnete, welchen eine primäre Erkrankung der Knochen, Knórpel, Liganente und Aponeurosen zum Grunde liegt. Ich habe diese vielmehr in allen Formen beobachtet. Allein die Erfahrung hat mich eben gelehrt, dafs das Vorkommen dieser Formen ungleich seltener ist, als das derjenigen, bei welchen die Veränderungen der passiven Bewegungsorgane secundärer Natur ist. In einer Abhandlung im Behrend, und Hildebrand'schen Journal für Kinderkrankheiten 1855. November- und December-Heft, habe ich dieses Verhältnifs für Scoliosis in einer statistischen Uebersicht nachgewiesen und Anhaltspunkte für die differentielle Diagnose aufgestellt. 
Aus den Dittel'schen Abhandlungen ist nun nicht recht ersichtlich, ob er seine Ansichten nur auf das vorliegende Präparat bezieht, oder ob er ihnen eine ganz allgemeine Geltung vindicirt. Doch mufs man das Letztere annehmen, wenn er bei Besprechung des Pes valgus l. c. S.417. sagt: „er könne sich nicht leicht denken, dafs irgend eine der wichtigen Veränderungen vom angeborenen Valgus als secundär zu betrachten sei; er glaube vielmehr annehmen zu müssen, dafs, ein $\mathrm{Mal}$ der Keim zur Deformität gegeben, sich diese in derselben ebenmälsigen Harmonie aller contribuirenden Theile ausbilde, wie es in der physiologischen Entwicklung der Fall sei. Nur der quantitative Unterschied sei in seiner Zunahme als secundär zu betrachten und zu bezeichnen. So sei die Verkürzung der Muskeln gewifs so primär, als die anomale Stellung im Fufswurzelgelenk und die tiefere Senkung der beiden Knöchel. Dagegen werde die Verfettung der Muskeln der Schlufspunkt jener Veränderung sein, die mil der ersten beharrlichen Verkürzung in der Organisation des Muskels beginne, so wie die Abschleifung und Bildung neuer Gelenke der Schlufspunkt jener Veränderungen sei, die mit der ersten Annäherung der Knochen beginne. Die Unterschiede zwischen primär und secundär treten also beim angeborenen Valgus nur als Kennzeichen des quantitativen Fortschrittes der Veränderungen auf."

Einen wesentlichen Unterschied aber zwischen angeborenem und erworbenem Valgus vermag ich aus der Dittel'schen Darstellung nicht herauszufinden. Es müfste denn der sein, dafs beim angeborenen „alle contribuirenden Theile" primär ergriffen sind, beim erworbenen das "combinirte Talo-calcaneonavicular-Gelenk", das ist aber eigentlich dasselbe. Denn zum combinirten Gelenk gehören am Ende auch die Muskeln. Dittel sagt darüber 1. c. S. 414.: „Das combinirte Talo-calcaneonavicular-Gelenk ist es also, aus welchem die abnorme Stellung des Valgus ausgeht und dadurch zu Stande kommt, dafs die Bewegung des Fufses in diesem Gelenke über das normale Maafs in allmähliger Steigerung hinausschreitet, und eben so 
kommen, wie gezeigt worden, die anderen Veränderungen zu Stande."

Dittel nimmt also beim angeborenen Valgus einen gegebenen Keim zur Deformität an und beim erworbenen eine Drehung, d. h. er verfällt in denselben Fehler, den er l. c. S. 412. an anderen Autoren rügt, indem er eine Erklärung der pathologischen Veränderungen giebt, „mit Worten, die dasselbe sagen, was sie erklären sollen."

Ich sehe aber auch die Nolhwendigkeit gar nicht ein, einen pathogenetischen Unterschied aufzustellen zwischen angeborenem und erworbenem Pes valgus, Pes equinus u. s. w. Meine Ansicht ist, dafs hier wie dort die Deformität primär bedingt sein kann durch ein Leiden des activen oder des passiven Bewegungsapparates. $\Delta$ ber auch bei der angeborenen Deformität ist gewils der in der oben angegebenen Weise gestörte Muskelantagonismus bei Weitem die häufigere Ursache, sei es nun, dafs dieser bedingt sei durch eine zufällige Lagerung, also eine local und peripherisch gestörte Muskelinnervation, oder durch eine solche von einem der Nervencentra ausgehende. Als Beweis für die Richtigkeit dieser Ansicht führe ich wieder an, dafs es auch bei der angeborenen Deformität meistens lange Zeit möglich isi, passiv die normale Stellung zu bewirken, was doch nicht möglich wäre, wenn bereits die Knochen, Ligamente etc. wesentliche Formveränderungen eingegangen wären. In der geringeren Anzahl von Fällen, wo dies nicht möglich ist, sind entweder bereits secundäre Veränderungen im passiven Bewegungsapparate eingetreten, oder dieser ist der primäre Sitz eines Krankheitsprozesses, dessen Ermittelung hier um so gröfseren Schwierigkeiten unterliegt, als jeder Anhalt einer Anamnese mangelt.

Bei-dieser Annahme ergielt sich nun in ungezwungener Weise, dafs die der Deformität zugehörigen, nicht muskulären, pathologischen Veränderungen im tendinösen, ligamentösen, knorpeligen und knöchernen Apparate als durch jene perverse Muskelthätigkeit bedingte secundäre Zustände zu betrachten sind.

Archiv f. patbol. Aaat. Bd. IX. Heft 4. 
So werden die Sehnen der an der Convexität gelegenen Muskeln nothwendig mit afficirt von der verminderten Innervation ihrer Muskeln. Daher erscheinen sie durchweg gedehnt, dünner, schmäler und schlaffer als normale. Dagegen sind die an der concaven Curve gelegenen, den Anfangs gesunden, aber verkürzten Muskeln angehörigen Sehnen verkürzt und dicker als normale. Je nach der Dauer ihres gezwungenen verkürzten Zustandes muls sich nothwendig ihre Textur verändern; ihre Verkürzung wird ebenso eine organische, wie die der Muskeln.

Dieselben Verhältnisse walten in den Ligamenten ob.

Die in antagonistischem Verhältnils zu den primär geschwächten oder gelähmten Múskeln stehenden Muskèln verkürzen sich automatisch und bewirken so die Drehung des Fufses um seine Queraxe. Beim Valgus sind dies die mit der Erhebung des äufseren Fufsrandes betrauten Muskeln. Der äufsere Fufsrănd rückt um so viel dem äufseren Knöchel näher, als der innere sich vom inneren entfernt. Der Kranke ist so genöthigt, beim Gehen mit dem inneren Fulsrande vorzugsweise den Boden zu berühren. Die Last des Körpers, im normalen Zustande auf das ganze Fulsgelenk gleichmärsig vertheilt, drückt nun vorzugsweise auf die Gegend des äufseren Knöchels und Fursrandes, während die entsprechende Gegend am inneren Fufsrande durch die Unthätigkeit der hier gelegenen Muskeln entlastet wird. Daher werden am äufseren Fulsrande zuerst die Knorpel und Knochen durch Druck resorbirt, während am inneren die Knorpel so weit atrophiren, als sie durch Verrückung der Gelenkflächen frei gelegt werden und dadurch aufhören ihrer Bestimmung zu dienen. Die aufgehobene Belastung der Knochen am inneren Fufsrande wird hier die Lockerung der Textur und Vergrölserung der Form zur Folge haben. Kurz es werden alle diejenigen Veränderungen eintreten, welche Dittel in seinen Präparaten vorgefunden und mit dankenswerther Treue beschrieben hat. Wir können diese Entstehungsweise der Veränderungen im knöchernen und knorpeligen Apparate der Deformitäten um so weniger bezweifeln, als wir im physiologischen, wie im pathologischen Zustande hinreichende 
analoge Erscheinungen beobachten. Wir sehen z. B. bei Tischlern liefe Aushöhlungen im Os sternum vom Drucke des dort eingesetzten Bohrers, und umgekehrt von innen nach aufsen sich entwickelnde Atrophie des Os sternum und der Rippen in Folge von Aneurysma aortae entstehen.

Anfangs beschränken sich diese secundären Veränderungen in der That nur auf die Lage der Knochen und Knorpel und Dehnung, respective Verkürzung der betheiligten Ligamente und Aponeurosen. Wäre dies nicht der Fall, so wäre es ja unmöglich, die normale Stellung passiv auch nur momentan herzustellen. Das ist aber so constant der Fall, dafs es mit Recht als ein wesentliches differenlielles Criterium zu betrachten ist von jener Gattung der Deformitäten, welchen bei ganz ähnlicher Form eine primäre Erkrankung der passiven Bewegungsorgane zum Grunde liegt.

Je weiter indels das Stadium der Deformilät von detm Beginne der Krankheit entfernt liegt, desto grölser müssen nothwendig alle jene Veränderungen in der Stellung, Form und Textur der Knochen, Knorpeln, Ligamente und Aponeurosen erscheinen. Daher kann nach 30jährigem Bestehen einer Deformität, wie der von Dittel beschriebenen, unmöglich erwartet werden, dafs es nach Durchschneidung der verkürzten Muskeln gelingen müsse, sofort die normale Form herzustellen. Ebenso únmöglich ist daraus, dals dies nicht sofort gelingt, zu folgern, dafs deswegen in den Muskeln nicht die primäre Bedingung zur Deformität liege. Wenn die Knochen so viele Jahre in einer deformen Stellung permanent verharrten, kann selbstverständlich die alienirle Muskelwirkung längst nicht mehr das allein zu beseitigende Moment abgeben. Im Gegentheil werden nunmehr die secundären Veränderungen ein nur sehr langsam zu überwindendes Hindernifs für die Herstellung der normalen Form darbieten. Hätte Ditlel, statt eines Valgus von 30 jähriger Dauer, einen nur kurze Zeit bestandenen zu präpariren gehabt, so hätte er sicherlich nichts von allen den umfangreichen Veränderungen in den Knochen und Knorpeln vorgefunden. Daher hat Brodie (Abhandlung über die Gelenk- 
krankheiten, übersetzt von Dr. Soer. 1853. S. 122.) nicht ganz Unrecht, wenn er sagt: ,die Bedeutung der pathologischen Anatomie, in so fern sie uns befähigt, tiefere Blicke in die Natur der Krankheiten zu werfen, kann nicht hoch genug angeschlagen werden. Doch hat sie nicht unter allen Umständen gleichen Werth. Die anatomische Untersuchung eines Gelenkes oder jedes anderen Organes in dem vorgerückten Stadium einer Krankheit kann uns über die ursprüngliche Natur der Krankheit und iiber das ursprünglich afficirte Gewebe wenig Aufschlufs geben u.s.f." Wenn es aber bei einer Deformität die Dauer allein ist, von welcher die vorhandenen Stellungs-, Form- und Texturveränderungen der passiven Bewegungsorgane erzeugt und weiter entwickelt werden, während die anomale Function der activen, d. h. der Muskeln, beim Beginne deutlich ausgesprochen ist, so liegt es wohl klar genug vor, dafs in diesen die primäre $\mathrm{U}$ sache und in jenen nur Folgeerscheinungen zu erblicken sind.

Für die Therapie kann diese pathogenetische Anschauung nicht anders als von grofsem Einfluls sein. Gleichwohl liegt es in der Natur der Sache, dafs diesèr nur ganz prägnant im ersten Stadium hervortritt. Im Allgemeinen stellen sich für alle musculären Gliederdeformitäten der hier besprochenen Art 2 Indicationen dar:

1) Kräftigung der an der convexen Curve befindlichen Muskeln durch alle dahin zielenden Mitlel. Unter diesen erachte ich die locale Electrisirung in Verbindung mit der localen Gymnastik nach Ling für die vorzüglichsten. Die erstere ist täglich 15 Minuten lang, die letztere täglich $2 \mathrm{mal} 1$ Stunde lang anzuwenden.

2) Verhütung und resp. Rückbildung der secundären Veränderungen in den passiven Bewegungsorganen. Dieser Indication entspricht die passive Zurückführung des Gelenkes und Gliedes in die normale Stellung und die kunstgemälse permanente Erhaltung desselben in dieser Stellung miltelst geeigneter Apparate.

Anders verhält sichs, wenn die Gliederdeformität eine ver- 
altete ist. Hier, wo die secundären Veränderungen des passiven Bewegungsapparates in der Regel der momentanen Herstellung der normalen Form widerstreben, ist für die Therapie als erste Indication die Beseitigung dieser secundären pathischen Zustände zu betrachten. Erst nachdem es gelungen ist, diese secundären Zustände so weit zu beseitigen, dafs die normale Form des Gliedes passiv momentan hergestellt werden kann, kann der nunmehrigen zweiten Indication, der Herstellung des gestörten Muskelantagonismus, in erwähnter Weise genügt werden.

Der ersten Indication genügen wir in den Fällen, wo die an der concaven Curve gelegenen Muskeln als Retraction sich darstellende Texturveränderungen eingegangen sind, durch die Tenotomie und nachherige mechanisch-orthopädische $\mathrm{Be}$ handlung. Ist es mittelsi dieser gelungen, die Rückbildung der in den passiven Bewegungsorganen bestandenen Texturund Formveränderungen auch nur annähernd zu bewirken, so tritt die Deformitäl für die Therapie in die Categorie des ersten Stadium. In diesem handelt es sich aber, wie ich oben ausgeführt, um die Herstellung der normalen Function des activen Bewegungsapparates, der Muskeln. Dies geschieht durch locale Electrisation und locale Gymnastik der an der Convexität gelegenen Muskeln. So lange dies Ziel noch nicht vollständig erreicht ist, bedarf es zugleich der entsprechenden Anwendung mechanischer Apparale, um die auf die Stellung des Gliedes deformirend einwirkende Last des Körpers unschädlich zu machen.

Diese für alle hier in Rede stehenden Gliederdeformitäten geltenden allgemeinen Prinzipien müssen mit Unsicht den individuellen Verhältnissen der Casuistik angepafst werden. Für die Deformitäten des Rumpfes stellen sich bei aller Analogie der pathogenetischen Momente für die gleiche Therapie doch abweichende Verhältuisse dar. Der Rumpf bietet nicht die gleichen günstigen Verhältnisse für die Application mechanischer Apparate dar, als die Extremitäten. Und wenn auch bei der immer mehr gesteigerten Vorvollkommnung der mechanischen Hülfsinittel momentan auf die Ausgleichung der Deformität hinge- 
wirkt werden kann, so treten doch ihrer permanenten Anwendung wichlige Bedenken entgegen. Der Rumpf birgt in sich die edelsten Organe. Welchen Nachtheil schon ein einfaches Corset übt, ist bekannt. Es unlerliegt daher keinem Zweifel, dals Apparate, wenn sie durch ihren mechanischen Druck und durch Extension vortheilhaft auf die Deformität wirken sollen, eine so beengende Befestigung erfordern, dafs sie einen gefährlichen Einflufs auf die Respiration, Blutcirculation und andere vitale Prozesse des Unterleibes ausüben müssen.

Ich wende dieselben daher bei den Deformitäten des Rumpfes nicht an, beschränke mich vielmehr auf die Anwendung der schwedischen Heilgymnastik täglich während zweier Stunden. Die Verschlimmerung aber der Deformität verhüte ich durch ruhiges Liegen der Kranken auf einem Planum inclinatum, welches ich je nach dem Grade des Uebels aufser der Nacht noch 4-5 Stunden des Tages innehalten lasse.

Diese Behandlungsweise gewährt mit Sicherheit folgende schätzbare Resultate:

1. Vor Allem die erfreulichste Beförderung des allgemeinen Wohlbefindens.

2. Die Verhütung jeder Steigerung der Deformität, in welchem Stadio dieselbe sich auch bereits befinden möge.

3. Vollkommene Heilung in den frïheren Stadien der Deformität, und wenigstens sehr oft noch eine erhebliche Besserung in vorgeriuckteren Stadien. 